\title{
Computer Eye-Tracking Model to Investigate Influence of the Viewer's Perception of the Graphic Information
}

\author{
Ekaterina Borevich ${ }^{1}$, Serg Mescheryakov ${ }^{1}$ and Victor Yanchus ${ }^{1}$ \\ ${ }^{1}$ Peter the Great St. Petersburg Polytechnic University, Polytechnicheskaya, 29, St.Petersburg, 195251, Russia
}

\begin{abstract}
The goal is to study the visual perception of graphic composition of various styles. An original author's method of conducting an experiment has been developed, which includes the preparation of stimulus material, collecting data, and statistical algorithms to analyze parametric data. The stimulus materials were based on graphic images in the cubism and abstractionism styles as well as on photorealistic images. An eye-tracking equipment was used to record eye movement activity and collect experimental data. The statistical analysis of the parametric data of the observer's viewing pattern has revealed that the viewer's perception of visual information is more effective by observers with art education. The results are of importance for developing effective training and test systems for operators, users, GUI developers, etc.
\end{abstract}

\section{Keywords}

Human visual perception, stimulus, eye-tracker, statistical treatment, abstract composition, art education

\section{Introduction}

Research using eye-tracking equipment has changed significantly as a result of technological progress [1]. Currently, high-tech hardware and software systems are used in a wide range of research [2]. The eye-tracking technologies have increased the efficiency of scientific work to study the perception of visual information [3]. One aspect of such research is to study the influence of the compositional construction of a visual information on its perception by the viewer. While another aspect is to study of the influence of the elements of a film frame on its perception by the viewer. Both aspects are important due to the limited demonstration time of a film frame. There is limited data on the influence of styled graphic compositions on the viewer's visual perception. Present work begins to fill in this gap demonstrating valuable practical data.

\section{Theoretical model}

A film frame is a structural unit of a film. It carries a visual component of a film as well as its semantic load. We define the visual appeal of a film frame as a property of a film frame, where frame elements are aimed at attracting and retaining the viewer's attention (Fig. 1) [4].

Zheleznyakov V.N. describes the visual perception of a film frame [5]. He describes a nervous rise, which is the main stimulus of perception. The nervous rise occurs in three directions. Each of them contains several groups of variables: psychophysiological, environmental, and comparative [5]. The composition of the frame and its color scheme have effect on the visual perception of a motion picture, because of the psychophysiological variables of visual perception [6].

GraphiCon 2021: 31st International Conference on Computer Graphics and Vision, September 27-30, 2021, Nizhny Novgorod, Russia EMAIL: plasma5210@mail.ru (E. Borevich); serg-phd@mail.ru (S. Mescheryakov); victorimop@mail.ru (V. Yanchus) ORCID: 0000-0001-6263-3901 (E. Borevich); 0000-0002-6748-1721 (S. Mescheryakov); 0000-0001-7220-0819 (V. Yanchus)

(c) (i) 2021 Copyright for this paper by its authors.

Use permitted under Creative Commons License Attribution 4.0 International (CC BY 4.0).

CEUR Workshop Proceedings (CEUR-WS.org) 


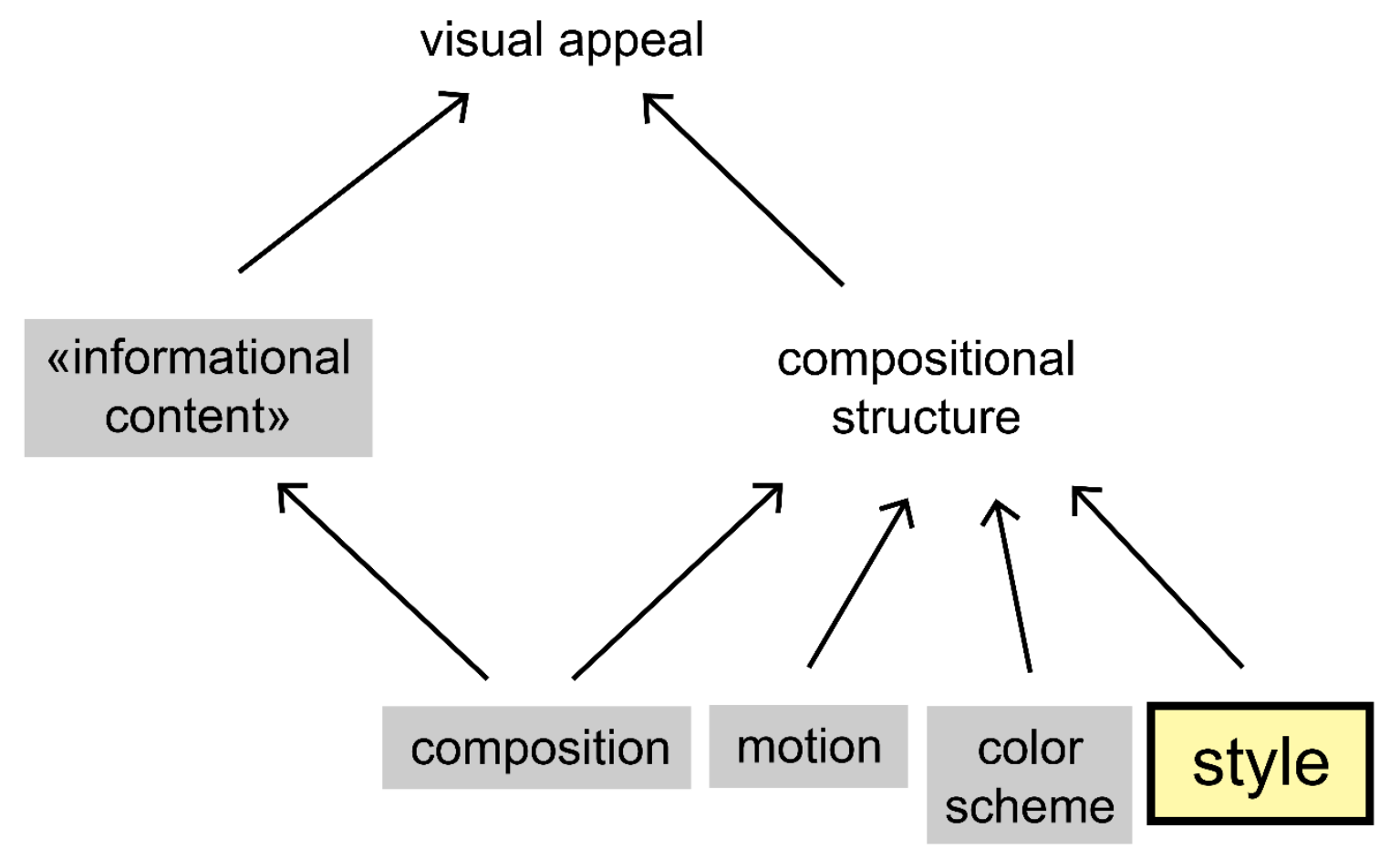

Figure 1: Schematic of the visual appeal of a film frame

In a series of experiments, we have established the statistically significant effect of the color scheme of the frame on its viewer's perception [4]. In subsequent work we studied the influence of image stylization on the viewer's perception of a film frame. Two groups of stimuli were studied: photorealistic stimulus material and animation stimuli [7]. No statistically significant effect of image stylization (photorealism-animation) was revealed presumably due not sufficient sensitivity of the experimental setup. However, the viewing pattern of animation frames was slightly different than that of the photorealistic one.

Ostrikov S.V. has discussed the problem of image stylization as an artistic method and described the main theoretical provisions [8]. The analysis of the creative products made by artists, designers, and animators made it possible to identify the phenomenon of stylization and describe the main techniques. Stylization is the reproduction or "imitation" of the figurative system and formal features of one of the styles which is used in a new artistic context [9].

Researchers has analyzed the influence of stylization on the perception of graphic information in various fields of activity:

- $\quad$ stylization of modern posters has increase the effectiveness of its impact [10];

- $\quad$ stylization of figured diagrams has increased the quality of information perception [11];

- $\quad$ stylization of comics has a role in the presentation of images [12].

In one study carried out using the eye-tracking technology, the dependence of the viewer's attention on the stylization degree of simplest geometric shapes was identified [13].

In this work, we carried out an experimental study of the influence of a graphic image stylization on viewer's perception. The stimulus materials were developed based on graphic images in the style of cubism and abstractionism, as well as photorealistic images. There were selected observers for participating in the experiment with art education and without art education.

\section{Experiment}

Stationary eye-tracking equipment, SMIRED-250 (Fig. 2), was used to record eye tracking activity. A methodology for conducting an experiment was developed, including the preparation of stimulus 
materials, task statements for observers, and an algorithm for statistical processing of the experimental data [7].

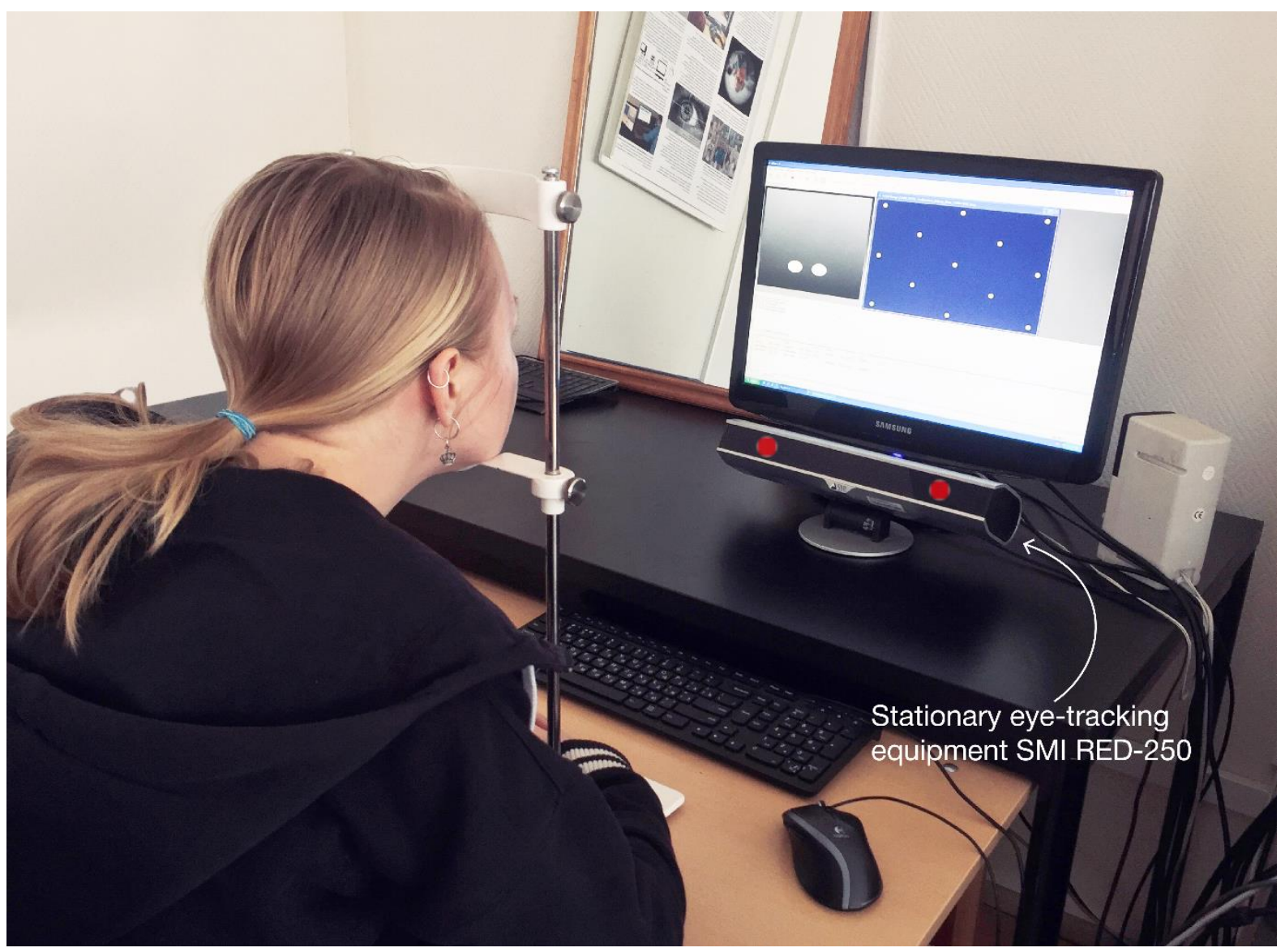

Figure 2: Stationary eye-tracking equipment

\subsection{Preparation of stimuli material}

The preparation of the stimulus material plays an important role in the methodology of the experiment, since it is the stimulus material that sets the basic conditions for the planned research. The stimulus materials were created based on graphic images in the style of cubism and abstractionism, as well as photorealistic images. Compositions mainly included abstract spots and simple geometric shapes such as point, line, and plane. All compositions, except for photographs, are non-objective, however, they have a meaning. All images were inscribed in a square with a size of $1080 * 1080$ pixels, and the background was in neutral gray color (Fig. 3).

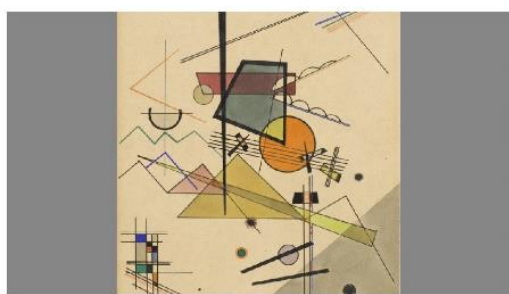

abstractionism

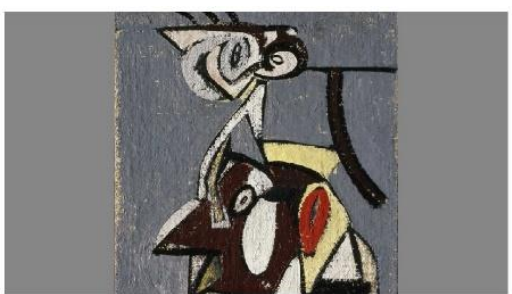

cubism

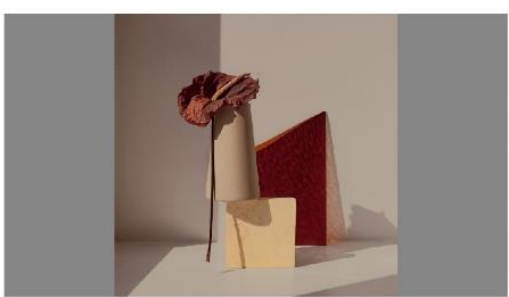

photorealism

Figure 3: Prepared stimulus material 


\subsection{Experimental Technique to collect experimental data}

Before the start of the experiment, an observer was explained in detail his tasks during the experiment. The task for the observer was to memorize and recognize images. Calm and comfortable environment was created during the experiment. The computer chair was adjusted individually for each observer. The number of distractions in the room was minimized: no extraneous sounds, and neutral lighting.

The experiment consisted of two stages (Fig. 4). At the first stage of the experiment, the observer was shown 6 images: 2 of cubism, 2 of abstractionism and 2 of photorealistic style. The observer's task was to remember as many images as she or he can. The time to memorize the images memorization was not limited. The images were displayed in a random order. The observer was independently switching to the next stimulus to complete the task. After completion of the first stage of the experiment, a 30 minutes timer was set for each observer and the second stage of the experiment was initiated.

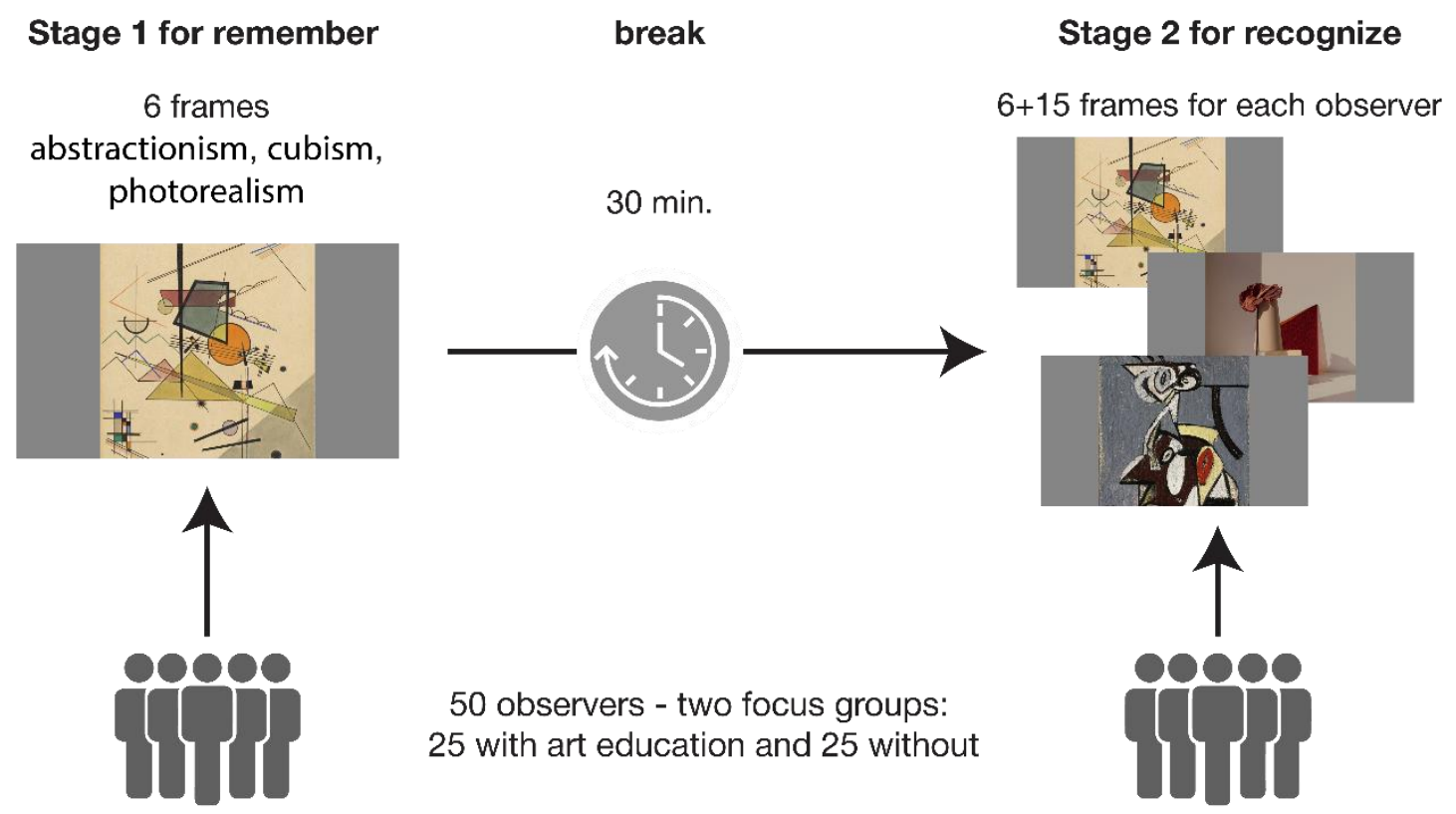

Figure 4: Experiment technique scheme

In the second stage, 15 more stimuli were added. Five stimuli from each group: cubism, abstractionism, photorealism. The stimulus material was played on the screen in random order. The task for the observer was to recognize if she or he has seen the stimuli or not and to answer "yes" or "no". The observer individually determined the time required for complete the task, after which she/he proceeded to the next stimulus.

\subsection{Statistical analysis of experimental results}

The 50 people have participated in the experiment. They were bachelor and master full time students 19 to 27 years year old. Everyone observer has normal vision. The observers were divided into two focus groups of 25 people each: a group with art education and a group without art education. Thus, the factor of the presence of art education was introduced into the experiment: 0 - observer without art education, 1 -observer with art education. Total number of fixations collected for each group was 6239 and 8435 respectively. Total of 14674 fixations were collected.

During statistical data analysis, the influence of the stylization factor (style) and the demonstration factor (demo) were analyzed. The values of the "style" factor: a - abstractionism, c - cubism, p photorealism. The "demo" factor had two values: 1 - stimuli demonstrated at both stages, 2 - stimuli demonstrated only at the second stage. 
The first stage of statistical data processing included the validation of the tasks performed by each observer. In addition, the parametric data obtained during the experiment were validated [14].

Statistical processing of experimental data was performed using ANOVA.

The following parameters of the viewing template were analyzed:

- $\quad$ viewing time of stimulus (Time);

- $\quad$ the average duration of fixation when viewing one stimulus (FixDurAv);

- the number of fixations when viewing one stimulus (Fix Num);

- fixation time when viewing one stimulus (FixDurTot);

- $\quad$ time of saccades when viewing one stimulus (SacDurTot);

- $\quad$ the number of saccades when view one stimulus (SacNum);

- the average duration of saccades when viewing one stimulus (SacDurAv);

- the maximum duration of saccades when viewing one stimulus (SacDurMax).

\section{Results and discussion}

\subsection{Results at the first stage of the experiment}

The graph of the distribution density of the stimulus viewing time (image memorization) by the observers versus the image stylization factor for different values of the art education factor is shown in Figure 5.

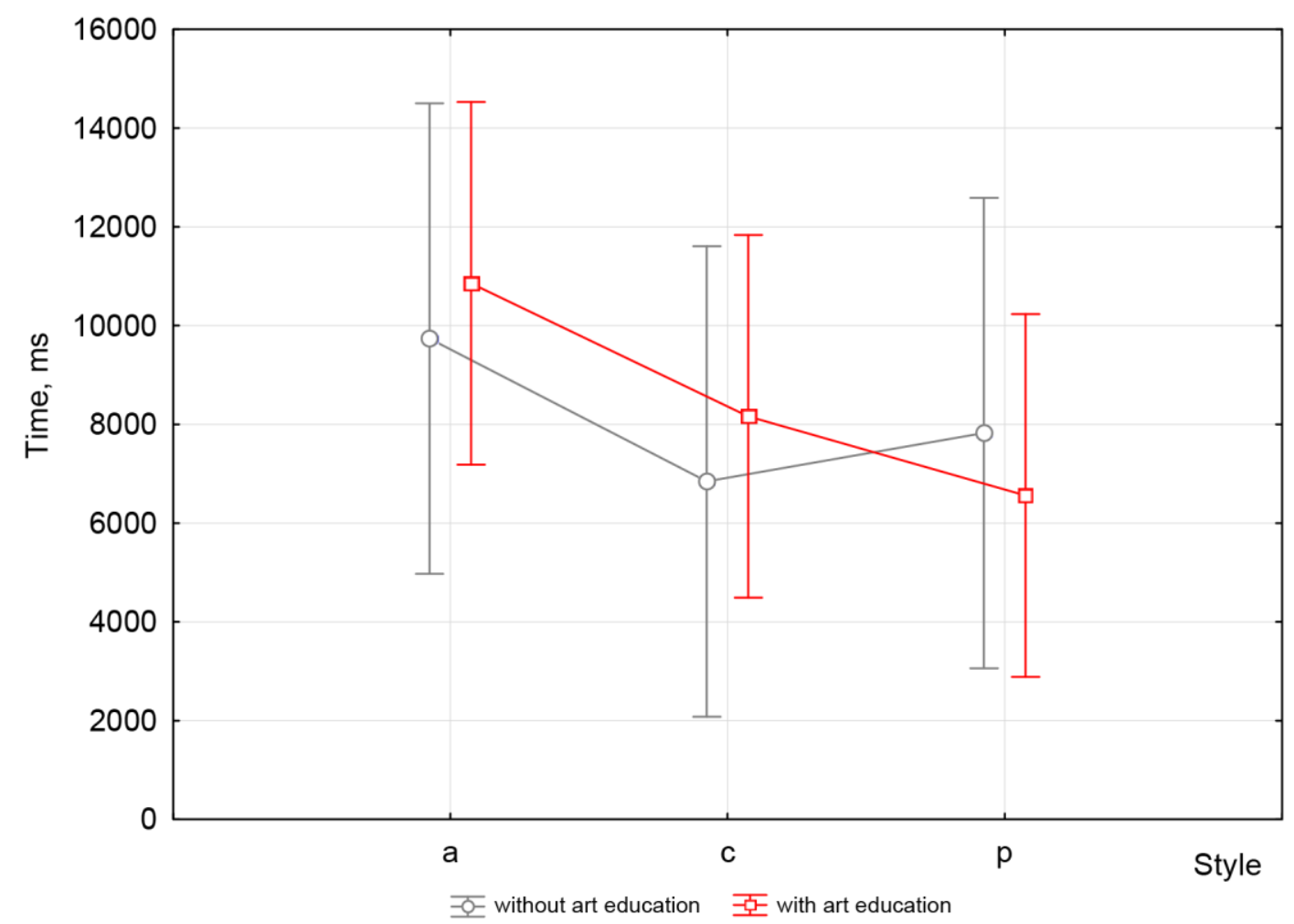

Figure 5: The graph of the distribution density of stimulus viewing time (image memorization) by the observers depending on the factor of image stylization for various values of the factor of art education at the first stage of the experiment

At the first stage of the experiment, mathematical processing of the data using analysis of variance ANOVA revealed that the parameters viewing time (Time), the number of fixations when viewing one stimulus (FixNum) and the number of saccades when viewing one stimulus (SacNum) do not have a statistically significant dependence on the stylization factor (style). However, there was a statistically significant effect of the presence of art education on the viewing pattern (Figure 6). 

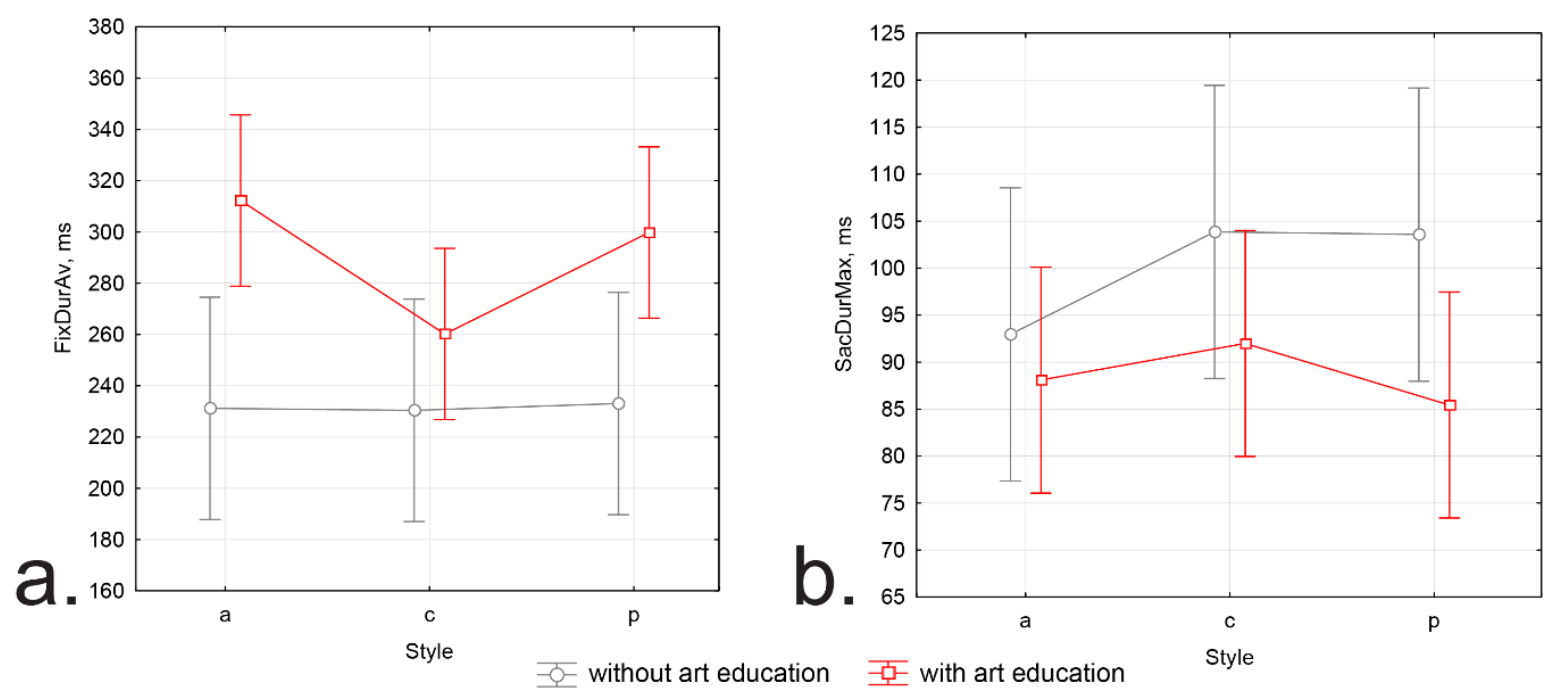

Figure 6: Graphs of the density distribution depending on the stylization factor for various values of the art education factor at the first stage of the experiment for: $a$ ) the average duration of fixation $b$ ) the maximum duration of saccades

The p-value criterion for assessing the effect of the presence of art education factor on the average fixation duration when viewing one stimulus was equal to 0.000271 , for the maximum saccadic duration when viewing one stimulus was equal to 0.0451 .

Experiment data suggest that the visual system by observers with art education makes longer fixations (Figure 6a) and shorter saccades (Figure 6b), when memorizing stimuli.

\subsection{Results at the second stage of the experiment}

The graph of the distribution density of the stimulus viewing time by the observers depending on the image stylization factor (style) for different values of the presence of art education and the demonstration factor (demo) at the second stage of the experiment (image identification) is shown in Figure 7.

At the second stage of the experiment, statistical processing of the data through analysis of variance ANOVA revealed that the parameters viewing time (Time), the number of fixations when view one stimulus (FixNum), the number of saccades when view one stimulus (SacNum) have a statistically significant dependence on the factor of stylization (style) and the factor of the presence of art education. The demo factor does not affect the parameters of the viewing template. The values of the p-value criterion are presented in Table 1.

Table 1

Values of the significance criterion $p$-value

\begin{tabular}{cccc}
\hline$p$-value & Time & FixNum & SacNum \\
\hline Style & $2.417 \mathrm{E}-06$ & $5.88 \mathrm{E}-09$ & 0.000638 \\
Art education & 0.0218 & 0.0422 & $7.56 \mathrm{E}-06$ \\
Demo & 0.771 & 0.557 & 0.311 \\
\hline
\end{tabular}

Figure 8 shows the graphs of the distribution density of the parameters of the stimulus consideration template depending on the image stylization factor (style) for various values of the factor of the presence of art education at the second stage of the experiment. 


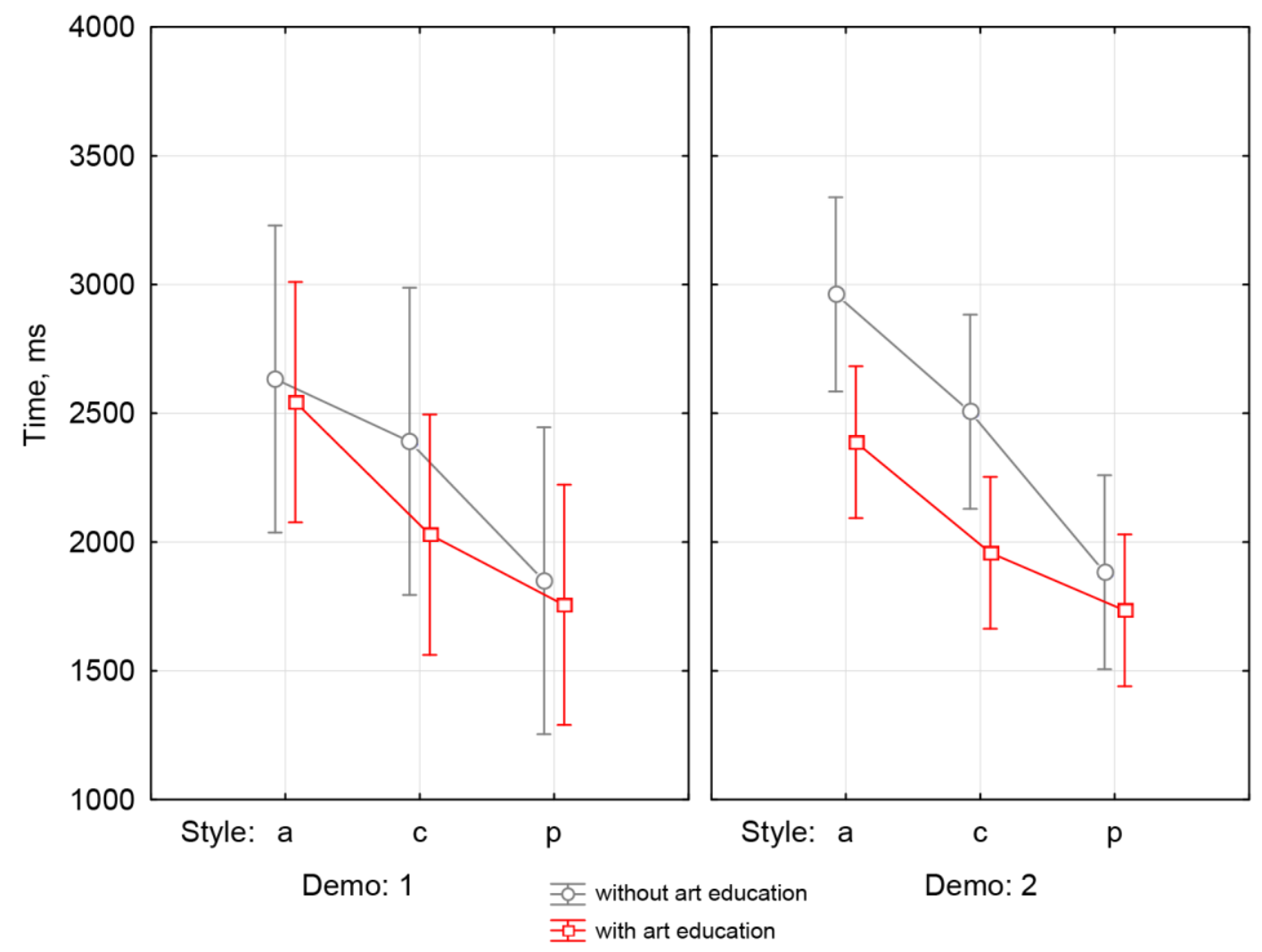

Figure 7: Graph of the distribution density of the stimulus viewing time by the observers depending on the image stylization factor (style) for different values of the art education factor and the demonstration factor (demo) at the second stage of the experiment

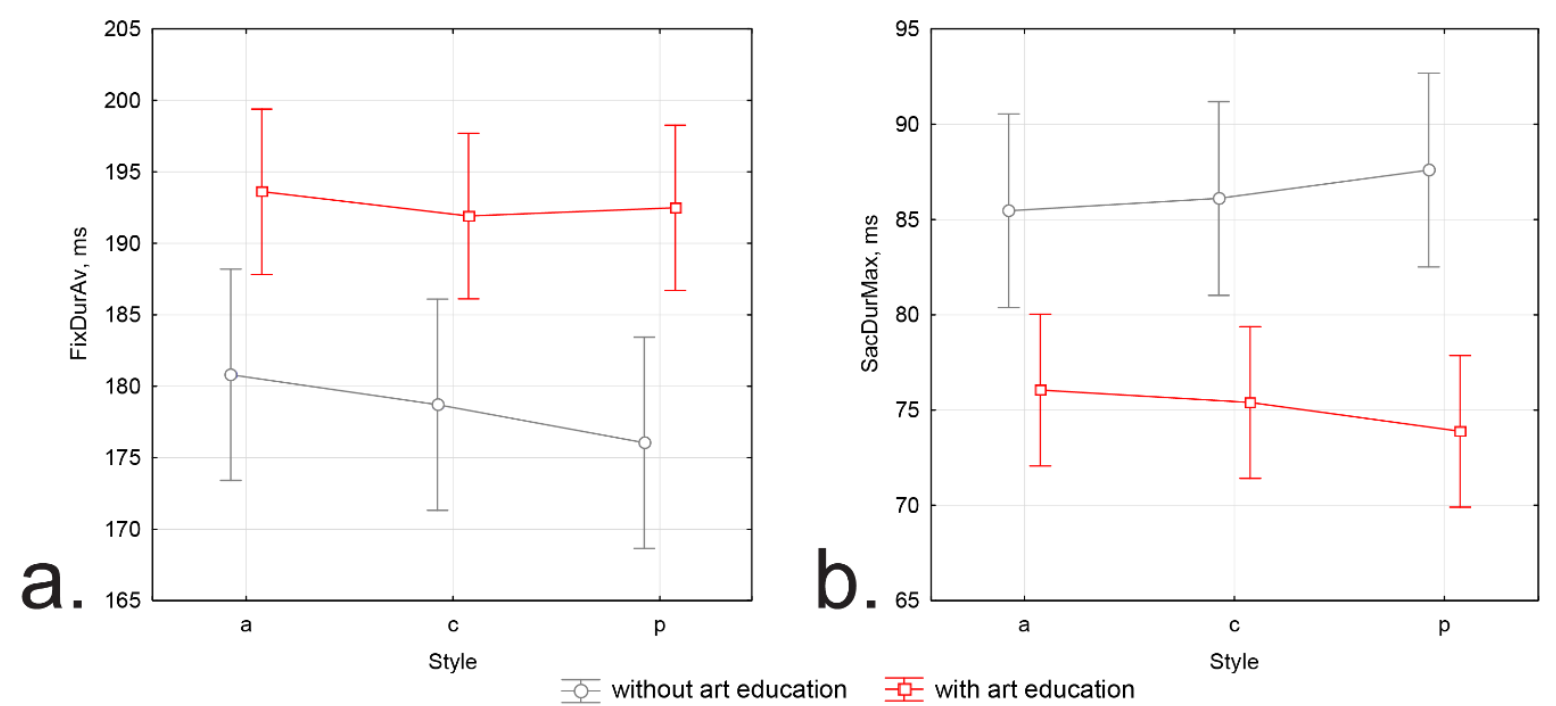

Figure 8: Graphs of the density distribution depending on the stylization factor for various values of the art education factor at the second stage of the experiment for: a) the average duration of fixation b) the maximum duration of saccades

Analyzing the graphs presented, it can be argued that the visual system by observers with art education makes longer fixations (Fig. 8a) and shorter saccades (Fig. 8b), when identifying stimuli, as in memorization. 


\subsection{Analysis of the Results}

Results of statistical analysis of experimental data point to the following:

1. The parameters of the viewing template depend on the task performed by the observer. The task of memorizing the stimulus material (Fig. 5) is completed by the observers four times longer than the task of identifying the stimulus (Fig. 7).

2. The parameters of the viewing template do not have a statistically significant dependence on the stylization factor when solving the task of memorizing stimulus material (Fig. 5).

3. The factor of image stylization has a statistically significant effect on the parameters of the stimulus material viewing template when solving the task of stimulus identification at the second stage (Table 1).

4. The factor of the presence of art education has a statistically significant effect on the parameters of the stimulus material viewing template when solving the task of stimulus identification at the second stage (Table 1).

5. The parameters of the stimulus viewing template during identification (second stage) do not depend on the demonstration factor (demo). It does not affect the performance of the task at the second stage that the observer saw the stimulus at the first stage (Table 1).

\section{Conclusion}

Presented work allows us to conclude that:

1. The human visual information perception system depends on the performed task. Memorization takes 4 times longer than recognition.

2. The system of perception of visual information works more efficiently for observers with artistic education. We observe statistically significant longer fixations and more saccade durations while maintaining the stimulus consideration total time. Can assumed that such observers read more data per unit of time. This fact does not speed up the process of solving the task memorizing stimuli. The observers with artistic education solve task of identifying faster.

3. In the experiment the observers spend more time solving the task of identifying when viewing stimuli with abstract composition. This is due to the visual system's need to process more information.

Based on the experimental results presented in this work, the following areas of research are suggested: development of a testing system (with the development of the proposed stimulus material) to identify students with creative abilities, to identify the opportunities for teenagers and their future professional orientation.

The results of the research are advisable to use for the development of educational programs on information design. Also, the results of the experiments are relevant in the development of intelligent interfaces for symbiotic control systems for remote dynamic objects, as well as in the development of training programs for operators of such systems.

The system of perception of graphic information works more efficiently on the observers with art education. This is due to specific professional orientation. We can assume that a similar effect can be seen on specialists in other fields. The development of the interfaces of computer systems should take into account the professional orientation of the user.

\section{References}

[1] L. Yarbus, The role of eye movements in the process of vision, Moscow, Nauka, 1965.

[2] V. A. Drummers, M. M. Milad, Oculography method in the study of cognitive processes and activities, Moscow, IP RAS, 1994.

[3] P. A. Orlov, V. V. Laptev, V. M. Ivanov, On the issue of using eye tracking systems, Scientific and technical statements of SPbSPU. Computer science. Telecommunications. Control, $\mathrm{SPb}$, Publishing house of SPbSPU, No. 5 (205), S. 8292 (2014) 82-92. doi: 10.5220/0005692102030211 
[4] E. V. Borevich, S. V. Meshcheryakov, V. E. Yanchus, Effective methods and models of digital processing of film material, Graphicon-2017: tr. 27th Intern. conf. on computer graphics and machine vision. Perm: Perm State Research University, No. 27 (2017) 51-54.

[5] V. N. Zheleznyakov, Color and contrast: Technology and creative choice, Moscow, VGIK, 2001.

[6] M. Chirimuuta, F. A. A. Kingdom, The Uses of Color Vision: Ornamental, Practical, and Theoretical, Minds \& Machines, Vol. 25(2) (2015) 213-229. doi: 10.1007/s11023-015-9364-z.

[7] S. V. Mescheryakov, V. E. Yanchus, E. V. Borevich, Statistical Model of Computing Experiment on Digital Color Correction, in: V. Vishnevskiy, K. Samouylov, D. Kozyrev (Eds.), Springer, Cham, LNCS 11965, Vol. 22 (2019) 140-150. doi: 10.1007/978-3-030-36614-8_11.

[8] S. V. Ostrikov, Stylization as a creative method of designing information graphic design, Bulletin of KSU im. N.F. Katanova, No. 6 (2013) 45-49. URL: https://cyberleninka.ru/article/n/stilizatsiya-kaktvorcheskiy-metod-proektirovaniya-dizaynerskoy-informatsionnoy-grafiki

[9] A. M. Kantor, Apollo. Fine and decorative arts. Architecture. Terminological dictionary, Moscow Research Institute of Theory and History of Fine Arts of the Russian Academy of Arts, 1997.

[10] P. A. Kudin, B. F. Lomov, A. A. Mitkin, Psychology of perception and the art of the poster, Moscow Plakat, 1987.

[11] P. A. Orlov, T. Ermolova, V. V. Laptev, A. Mitrofanov, V. M. Ivanov, The Eye-tracking Study of the Line Charts in Dashboards Design, Imaging and Computer Graphics Theory and Applications, Vol. 2 (2016) 205-213. doi: 10.5220 / 0005692102030211.

[12] A. L. Miklashevich, Stylization in comics and its impact on perception, 18th All-Russian Scientific and Practical Conference of Postgraduates, Masters and Students "Creativity of the Young: Design, Advertising, Information Technologies", Omsk (2019) 45-49.

[13] S. P. Elshansky, Preference and ignoring of simple geometric shapes in the process of their visual perception (eye tracking follow), Modern scientific research and innovation, №4, Part 2, 2014.

[14] E. V. Borevich, S. V. Meshcheryakov, D. A. Schemelinin, V. E. Yanchus, Methods and algorithms for experimental research of graphic models of color solutions, Software systems and computational methods, No. 4 (2018) 144-153. doi: 10.7256/2454-0714.2018.4.27695. 